\title{
Estimation of Real-time Blood Pressure during Motion Using Electrocardiography Waveform
}

\author{
Hsien-Wei Tseng, ${ }^{1}$ Yang-Han Lee, ${ }^{2 *}$ Chien-Da Huang, ${ }^{2}$ Yi-Lun Chen, ${ }^{2}$ and Yanfang Liu ${ }^{1}$ \\ ${ }^{1}$ School of Information Engineering, Longyan University, \\ No. 1 Dongxiao North Road, Longyan, Fujian, China \\ ${ }^{2}$ Electrical and Computer Engineering, Tamkang University, \\ 151, Ying-Chuan Rd., Tamsui, New Taipei City, Taiwan (R.O.C.)
}

(Received November 3, 2017; accepted March 7, 2018)

Keywords: electrocardiography (ECG), blood pressure, HRV, so and chan algorithm, biomedical electronics, wearable

Electrocardiography (ECG) is a safe, noninvasive test with a history of more than a hundred years of use. A physician can judge whether a person is healthy by virtue of the ECG waveform. According to statistics from the World Health Organization (WHO), cardiovascular disease (CVD) is number one among the ten major causes of death globally, and the probability of CVD increases with age for office workers with irregular lifestyles and for obese people. People know about their health because of physiological signals, and there is a close relationship between CVD and blood pressure. The most common method currently is self-health monitoring by measuring blood pressure with a home hematomanometer. However, it may be inconvenient to measure blood pressure with a traditional hematomanometer, and it may be especially difficult if blood pressure needs to be measured under dynamic conditions. In this study we aimed to measure blood pressure with a wearable 2-point ECG device and to monitor the most obvious characteristics of QRS (ventricular depolarization) duration and change in blood pressure to enable health monitoring at any time.

\section{Introduction}

The heart is one of the most important organs of the human body, and electrocardiography (ECG) currently provides the best evidence to judge its health. A doctor can assess whether people are healthy using the information it provides. The most significant measurement among various cardiovascular disease (CVD) signals is the QRS (ventricular depolarization) waveform. In this study, we calculated the correlation of the QRS duration to blood pressure using an improved ECG guiding measurement, in an effort to replace complex equipment and devices in hospitals with algorithms.

*Corresponding author: e-mail: yhleepp@gmail.com http://dx.doi.org/10.18494/SAM.2018.1821 


\section{Research Methods}

\subsection{ECG waveform}

When the heart contracts, a conducting strip pasted on the skin measures the electricpotential transmission of the heart. An ECG not only records electric-potential changes of a single heart chamber or atrium cell, but of the whole heart, via the QRS waveform. ${ }^{(1-7)}$ The electric depolarization of the heart chamber is normally less than $110 \mathrm{~ms}$, as shown in Fig. 1.

\subsection{Wavelet theory}

The initial standard tool for signal analysis and signal information acquisition is the Fourier transformation (FT), the conversion formula of which is

$$
F(\omega)=\int_{-\infty}^{\infty} f(t) e^{-j w t} d t
$$

However, Fourier conversion is a kind of average analysis whereas the scope of the analysis is the entire time span; therefore, if there is a large change in a particular time point (emergent signal), for example, $20 \mathrm{~Hz}$, when the occurrence time is unknown, short-time Fourier transform (STFT) is applied to obtain information from the signal frequency field and time field. However, this information cannot be analyzed accurately. In contrast, wavelet conversion has been applied to the decomposition of signals in recent years and provides flexible resolution in the time and frequency domains.

\subsubsection{Multiple wavelet resolution}

Wavelet conversion ${ }^{(8,9)}$ is applicable to the detection and monitoring of signal noise, while wavelet analysis and wavelet conversion show signals with vibration waves (called mother wavelets) of limited lengths or rapid decreases. The waveform is contracted or translated to match the input signal. The conversion equation for a continuous wavelet for any function $f(x)$

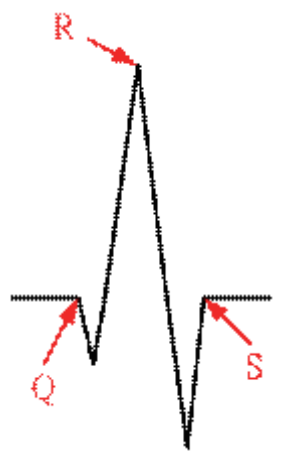

Fig. 1. (Color online) Schematic diagram for the QRS of an ECG. 


$$
C\left(2^{j}, b\right)=\frac{1}{\sqrt{2^{j}}} \int_{-\infty}^{\infty} f(t) \psi\left(\frac{t-b}{2^{j}}\right) d t
$$

where $\psi(x)$ is the mother wavelet of the wavelet function, and the mother wavelet $\psi(t)$ conforms to the following three conditions.

1. The function shall have vibration characteristics and the integral of the function shall be 0 , as defined by

$$
\int_{-\infty}^{\infty} \psi(t) d t=0
$$

2. The integral of the square of the function is a limited value and shall converge, as shown by

$$
\int_{-\infty}^{\infty}|\psi(t)|^{2} d t<\infty
$$

3. The function of the band communication signal decreases gradually to 0 towards infinity.

The term $C$ is the coefficient after wavelet conversion, $j$ is the contraction coefficient of wavelet conversion, $b$ is the horizontal coefficient for wavelet transform, and signals of different dimensions can be obtained depending on the value of $j$.

Figure 2 shows the multiple-level decomposition of multiple resolutions of wavelets to the original signal for different dimensions. The decomposition steps are as follows: the original signal of wavelet order is transformed to obtain cA1 of the low-frequency component and cD1 of the high-frequency component. Then cA1 is decomposed using wavelets to obtain cA2 and cD2. In this same way, we can obtain cAn and cDn for higher dimensions.

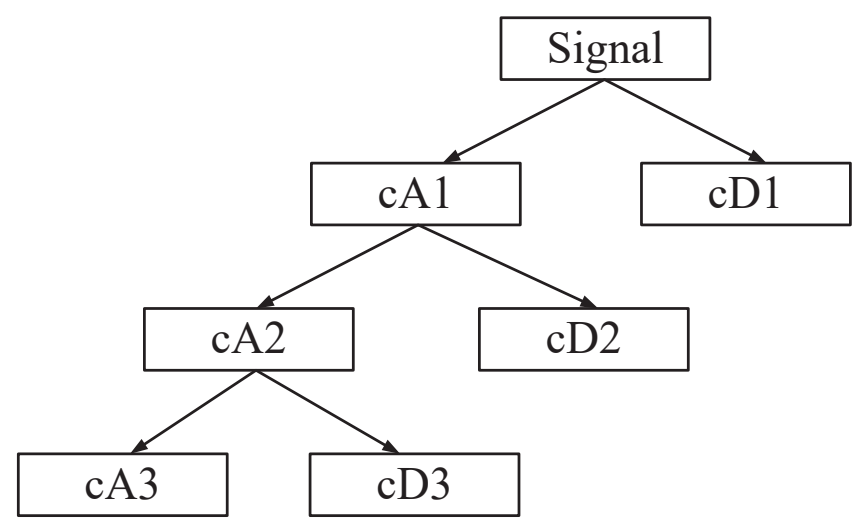

Fig. 2. Multiple resolution of wavelets for different dimensions. 


\subsubsection{Wavelet characteristics and nature}

The principle of wavelet conversion is similar to that of FT. The difference is that wavelet conversion has a mother function and a new function is taken as the base function. The signal is analyzed using the base function after extension and contraction of the mother function in a certain proportion.

Figure 3 is the schematic diagram of a filter for wavelet conversion and corresponds to wavelet conversion for multiple decompositions, in which high-frequency and low-frequency signals are decomposed. The signal in the figure is the original signal with noise, and signal D1 is the high-frequency component obtained using a high-pass filter, while signal A1 is the lowfrequency component obtained using a low-pass filter.

The mathematical formula of wavelet conversion is shown by Eq. (5), and $\psi(t)$ is the wavelet function, as shown by Eq. (6). The term $\overline{\psi_{a, \tau}(t)}$ is the conjugate complex of $\psi_{a, \tau}(t)$.

$$
\begin{gathered}
W_{\psi} f(a, \tau)=\int_{-\infty}^{\infty} f(t) \overline{\psi_{a, \tau}(t)} d t \\
\psi_{a, \tau}=a^{\frac{1}{2}} \psi\left(\frac{t-\tau}{a}\right), a>0
\end{gathered}
$$

Here, $a$ represents the coefficient for contraction or extension of $\psi(t)$, while $\tau$ is the signal translation to $\psi(t)$. Wavelet mother function bior 5.5 is adopted in this research, as shown in Fig. 4 , because bior 5.5 has the most similar characteristics to those of the ECG signal.

\subsection{Find $R$ wave position using So and Chan algorithm}

The most important characteristic of the QRS complex wave is wave R; therefore, wave R was selected by the So and Chan method. The process diagram is shown in Fig. 5. From the beginning, the initial value is calculated and the maximum value of the first 40 points (nearly

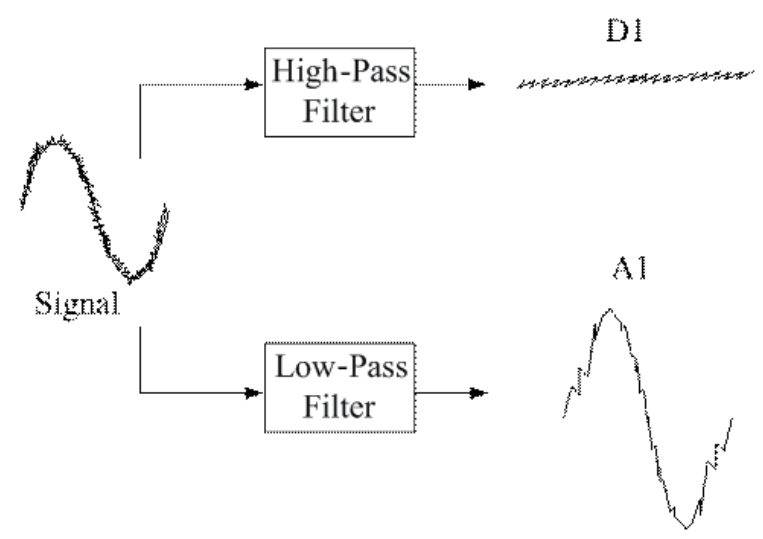

Fig. 3. Schematic diagram of an order I wavelet transform filter. 


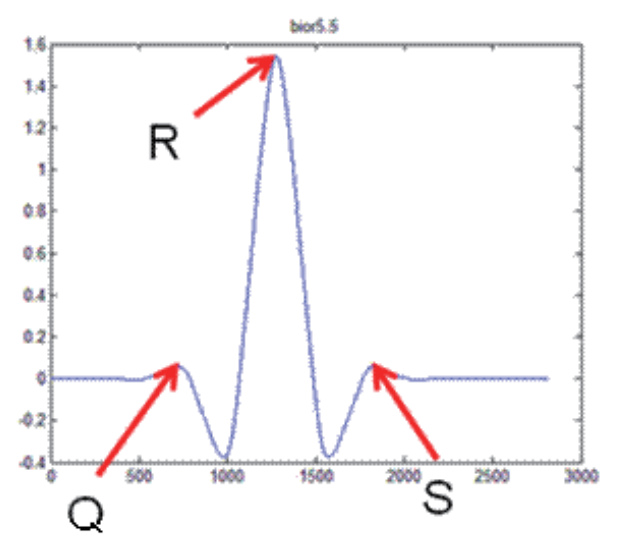

Fig. 4. (Color online) Wavelet mother function bior 5.5.

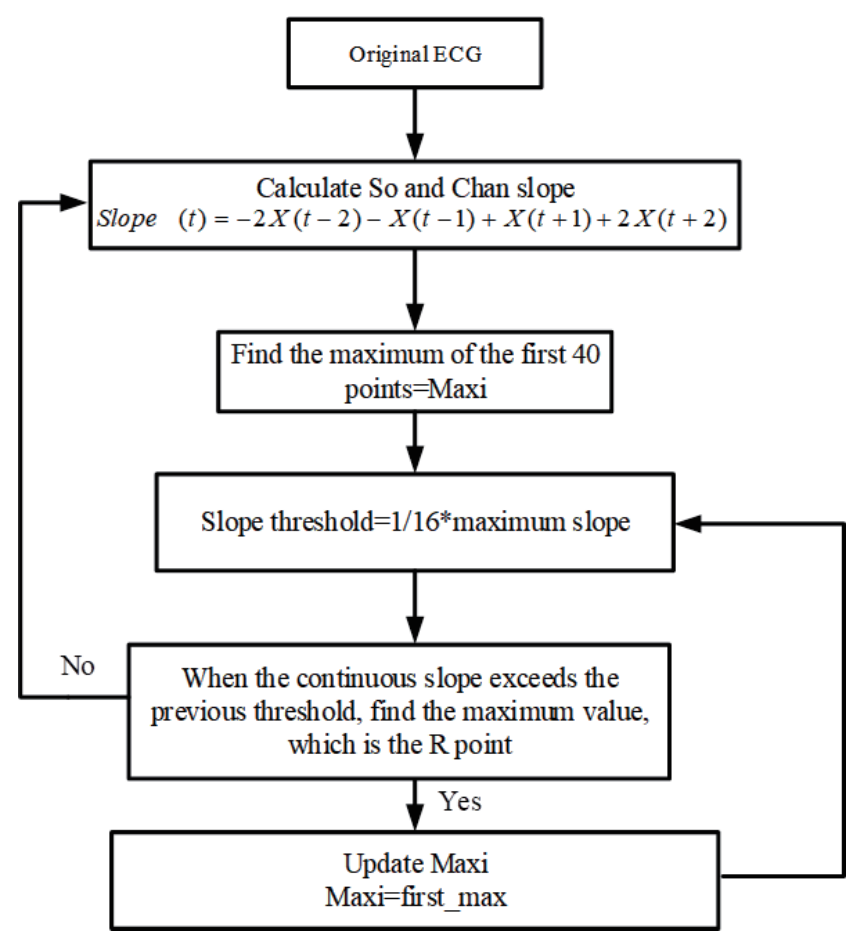

Fig. 5. Process diagram of So and Chan algorithm.

$160 \mathrm{~ms}$ ) is calculated. These 40 points are adjustable. Fewer points indicates a higher number of sampling times over the entire ECG. Accuracy is higher with more samplings, although the calculation time is longer. ${ }^{(10,11)}$

\subsection{Simulation diagram of So and Chan algorithm}

Figure 6 shows the original dynamic ECG signal; the sampling frequency is $200 \mathrm{MHz}$. There is much noise ${ }^{(12)}$ and the QRS characteristics are not significant. Therefore, the previous wavelet conversion theory was applied and the results of the analysis of the dynamic ECG for multiple resolutions are shown in Fig. 7 for the low-frequency part obtained by order-I wavelet conversion. Figure 8 shows the results for the high-frequency part obtained by order-I wavelet conversion. After order-I wavelet conversion, wave $\mathrm{R}$ is relatively obvious.

Figure 9 shows wave $\mathrm{R}$ obtained from the wavelet using the So and Chan algorithm. The position of point $\mathrm{R}$ is located and the forward and backward positions of slope turning points $\mathrm{Q}$ and $\mathrm{S}$ are sought.

\section{Research Results}

\subsection{ECG signal module}

In this study, the ECG signal is measured using BeneGear Inc. ECG125, as shown in Fig. 10. This module contains Bluetooth enabling transmission of the measured data to other devices. ${ }^{(13-17)}$ 


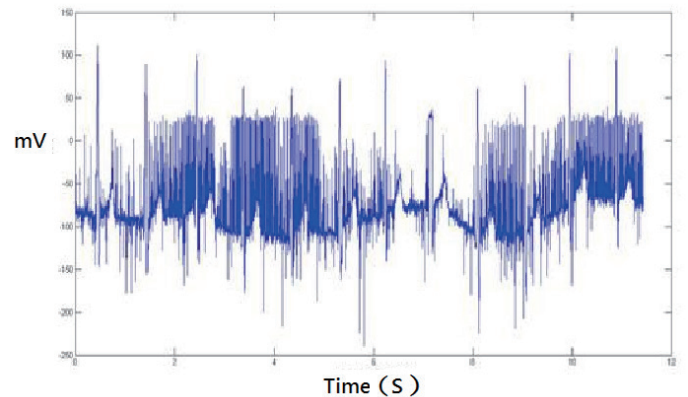

Fig. 6. (Color online) Original dynamic ECG signal.

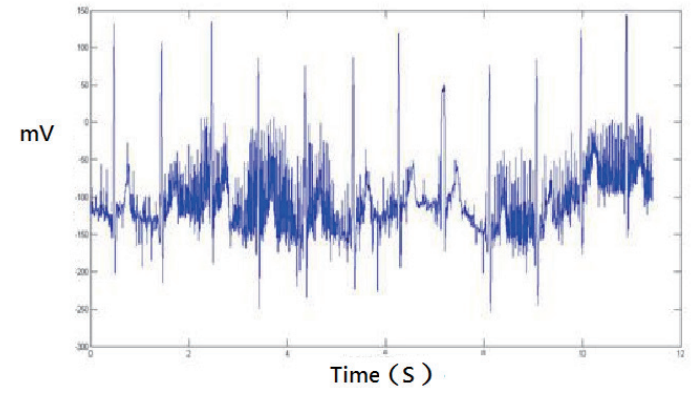

Fig. 7. (Color online) Low frequency in order-I wavelet conversion.

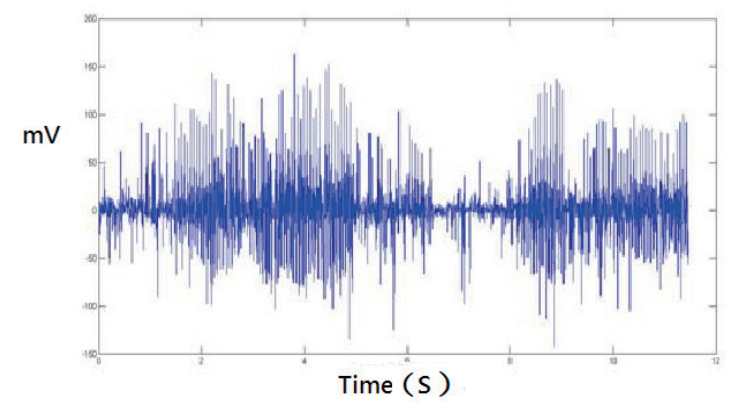

Fig. 8. (Color online) High frequency in order-I wavelet conversion.
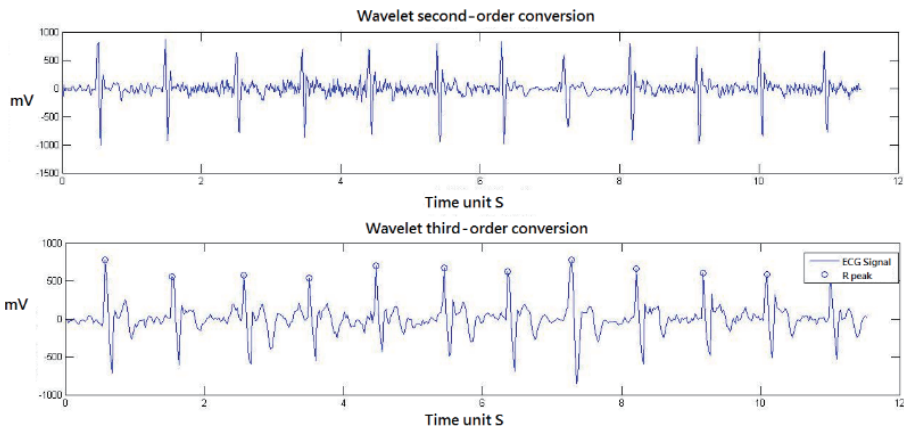

Fig. 9. (Color online) Position of point $\mathrm{R}$

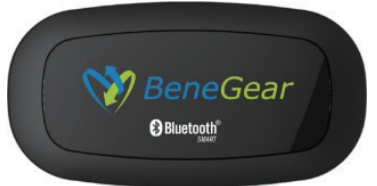

(a)

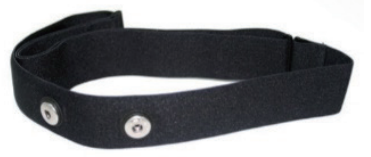

(b)

Fig. 10. (Color online) (a) BeneGear Inc. ECG125 and (b) heartbeat monitoring belt.

\subsection{Test of the blood pressure algorithm}

The test of the blood pressure algorithm is shown in Fig. 11. In this study, the test environment is divided into two different environments: dynamic measurement and static measurement. Participants wear a 2-point ECG device ${ }^{(18-23)}$ and a hematomanometer, and data are recorded and simulated. ${ }^{(24)}$ After comparing data, test methods are divided into the following four states. 


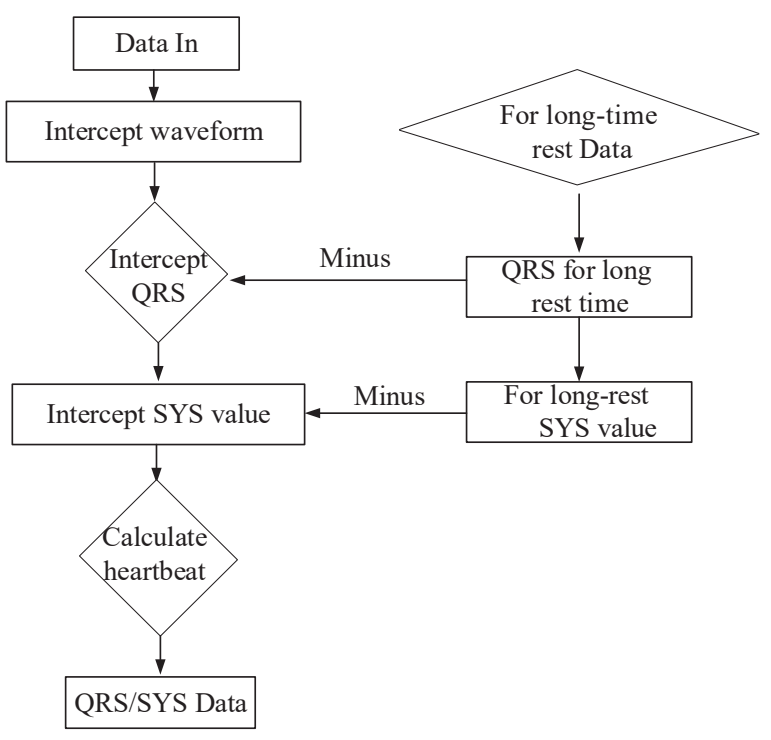

Fig. 11. Test of the blood pressure algorithm

1. State after a 5-min rest after running.

2. Quick running state: test time $752 \mathrm{~s}$.

3. General walking state: test time $809 \mathrm{~s}$.

4. Long-time sleeping state: test time $27900 \mathrm{~s}$.

Participants use treadmills to elevate their heart rate in the dynamic test environment. Blood pressure is measured within $30 \mathrm{~s}$ of ceasing running. Heartbeat waveform and blood pressure measurements are shown in Figs. 12 and 13, respectively.

An electric transmission system controlled by the nervous system in the human heart controls the heartbeat. When measuring static ECG, a comfortable sleeping environment is beneficial to maintain a regular heartbeat. The heartbeat of normal adults is $60-100$ times per minute at rest. Under normal daily activity, the heartbeat changes as activities change. For example, the heartbeat is more rapid during exercise or when a person is tense. During sleep, the heartbeat of a normal adult may decrease to 30-60 times per minute, so it is favorable to compare the differences between the static state and the dynamic state in a sleep environment.

\subsection{Heart rate QRS wave and actual measurements of blood pressure}

Figures 14-17 are waveform obtained in different test environments. Tables 1 and 2 are measured data. We analyzed the "prediction of blood pressure during exercise" using the data collected from static records of the measurements as examples. We obtained a mean value for the QRS of $-122.3 \mathrm{~mm}$ under a stable wave peak and found the QRS mean value for each dynamic section. Then, we compared the N-QRS values and obtained the estimated exercise blood pressure shown in Fig. 18. 


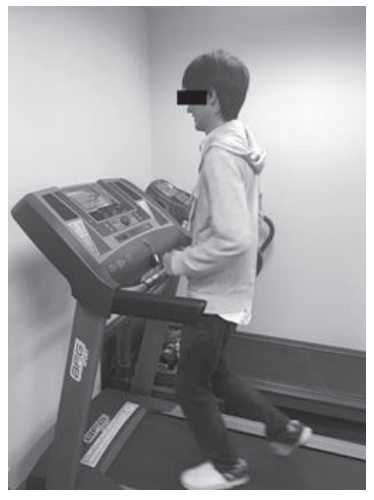

Fig. 12. Actual running measurements for dynamic ECG.

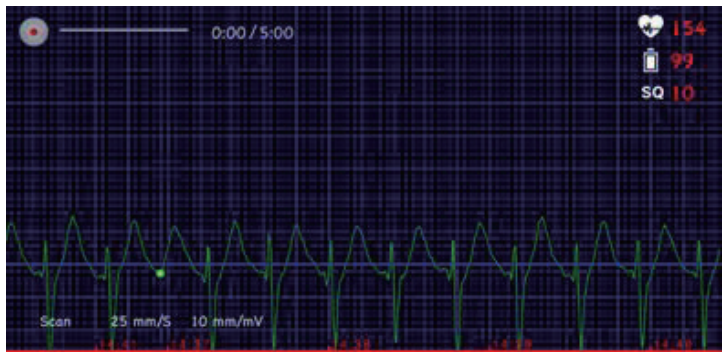

Fig. 14. (Color online) Waveform figures at the start of running.

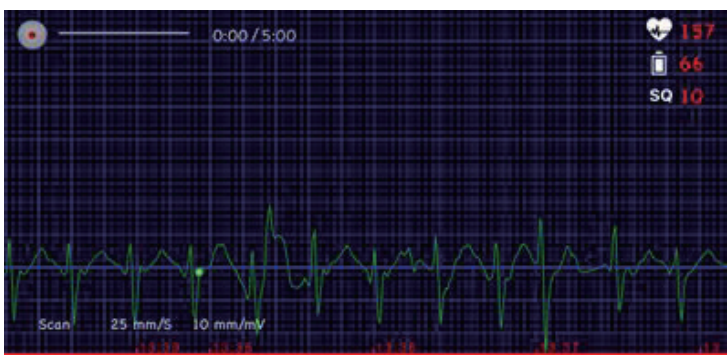

Fig. 16. (Color online) Waveform figures for second running interval with rapid running.

Table 1

Static measurement data.

\begin{tabular}{lcccc}
\hline Status & $\begin{array}{c}\text { PULSE } \\
(\mathrm{mm})\end{array}$ & $\begin{array}{c}\text { SYS } \\
(\mathrm{mmHg})\end{array}$ & $\begin{array}{c}\text { DIA } \\
(\mathrm{mmHg})\end{array}$ & $\begin{array}{c}\text { QRS } \\
(\mathrm{mm})\end{array}$ \\
\hline Normal-1 & 70 & 117 & 82 & 120.6 \\
Normal-2 & 62 & 112 & 71 & 122 \\
Normal-3 & 64 & 118 & 64 & 124.3 \\
Average & 65 & 116 & 72 & 122.3 \\
\hline
\end{tabular}

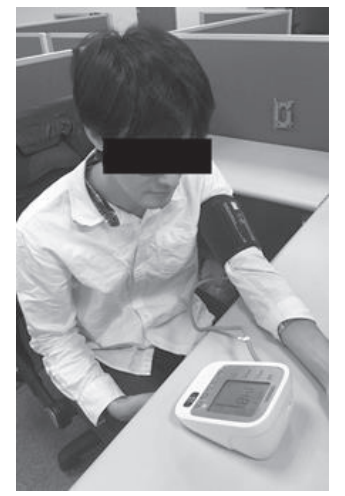

Fig. 13. Blood pressure measurement.

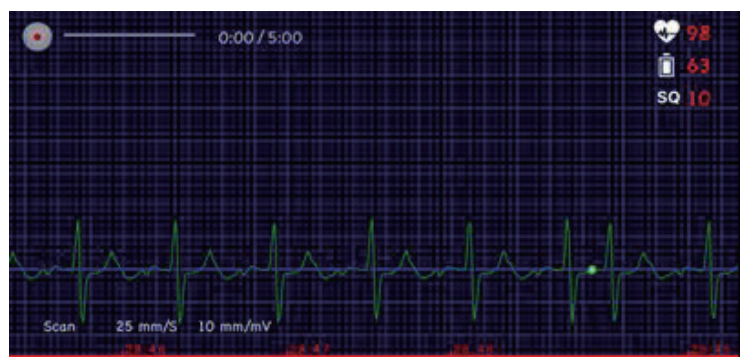

Fig. 15. (Color online) Waveform figures after $5 \mathrm{~min}$ of rest.

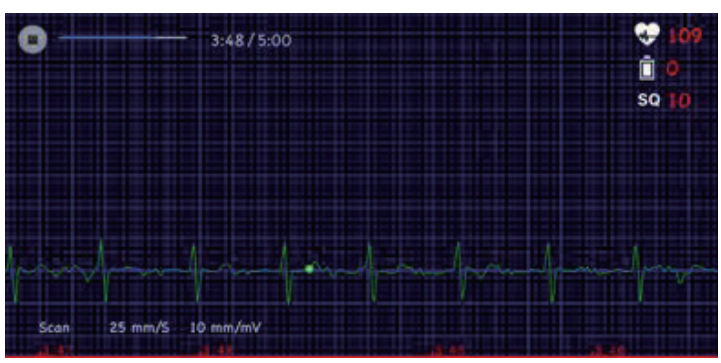

Fig. 17. (Color online) Waveform figures for walking.

Table 2

Dynamic energy measurement data.

\begin{tabular}{lcccr}
\hline Status & $\begin{array}{c}\text { PULSE } \\
(\mathrm{mm})\end{array}$ & $\begin{array}{c}\text { SYS } \\
(\mathrm{mmHg})\end{array}$ & $\begin{array}{c}\text { DIA } \\
(\mathrm{mmHg})\end{array}$ & $\begin{array}{c}\text { QRS } \\
(\mathrm{mm})\end{array}$ \\
\hline Walk & 105 & 126 & 60 & 110.5 \\
Run & 157 & 139 & 79 & 96.3 \\
Rest 5 min & 96 & 112 & 59 & 108.4 \\
Small run & 124 & 135 & 71 & 104.8 \\
\hline
\end{tabular}




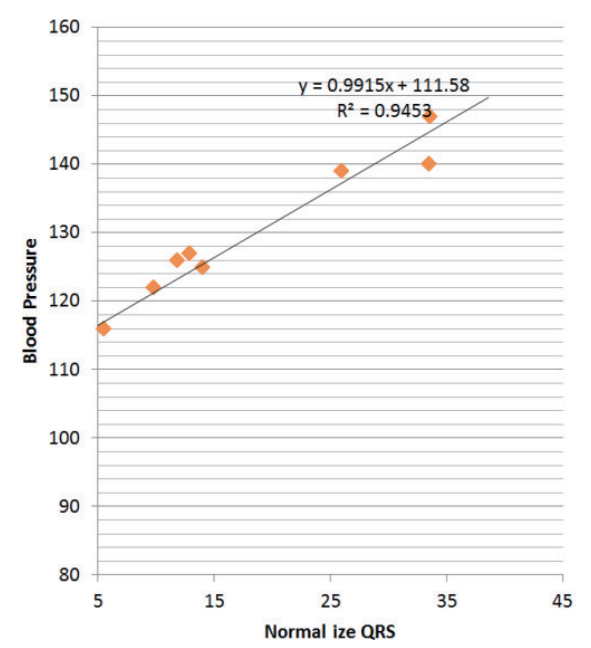

- N-QRS

Table 3

All measurement data.

\begin{tabular}{rcrcc}
\hline HR & SYS & QRS & Normal & N-QRS \\
\hline 105 & 125 & 108.3 & 122.3 & 14 \\
154 & 147 & 88.7 & 122.3 & 33.6 \\
98 & 116 & 116.8 & 122.3 & 5.5 \\
105 & 126 & 110.5 & 122.3 & 11.8 \\
157 & 139 & 96.3 & 122.3 & 26 \\
96 & 112 & 108.4 & 122.3 & 13.9 \\
109 & 127 & 109.4 & 122.3 & 12.9 \\
153 & 150 & 88.8 & 122.3 & 33.5 \\
99 & 122 & 112.5 & 122.3 & 9.8 \\
\hline
\end{tabular}

Fig. 18. (Color online) Estimated blood pressure during exercise.

\section{Conclusions and Future Study}

In this study, heartbeat and waveforms were measured by having participants wear an ECG rather than a traditional hematomanometer and the blood pressure during exercise corresponding to QRS intervals was estimated using static blood pressure and dynamic blood pressure values. For example, Eq. (7) can be used with the N-QRS value in Table 3 as $x$ to obtain the SYS contraction pressure.

$$
y=0.9915 x+111.58
$$

As seen in Fig. 18, we found a positive correlation of up to 0.9453 between the QRS wave and blood pressure during exercise. This may allow us to prevent the occurrence of CVD during exercise. Moreover, the measurements better reflect current blood pressure changes than do measurements using traditional static hematomanometers.

\section{Acknowledgments}

This research and work is supported in part by the Longyan Municipal Science and Technology Bureau, Fujian Province and Longyan University, through the Qi Mai Science and Technology Innovation Fund under contract 2017QM02; Education Scientific Research Project of Young Teachers of Fujian Province under Grant No. JAT170577; the Climbing Project of Longyan University under Grant No. LQ2015031; Fujian University's Key Laboratory of Big Data Mining and Application (Longyan University); the Collaborative Innovation Project of Longyan University (Zhang Ling); and TamKang University, Ministry of Science and Technology, Republic of China, under grant MOST 106-2221-E-032-003. 


\section{References}

1 X. Zhu, K. Yoshida, W. Yamanobe, Y. Yamamoto, W. Chen, and D. Wei: IEEE EMBS Asian-Pacific Conf. Biomedical Engineering (IEEE, 2003) 48-49.

2 Z. Ihara, V. Jacquemer, J. M. Vesin, and A. Van Oosterom: Computers in Cardiology (IEEE, 2005) 203-205.

3 R. R. Bond, D. D. Finlay, C. D. Nugent, and G. Moore: Computing in Cardiology (IEEE, 2010) 285-288.

4 S. Z. Mahmoodabadi, A. Ahmadian, M. D. Abolhasani, M. Eslami, and J. H. Bidgoli: IEEE-EMBS 2005. 27th Annu. Int. Conf. Engineering in Medicine and Biology Society (IEEE, 2006) 3902-3905.

5 G. M. Friesen, T. C. Jannett, M. A. Jadallah, S. L. Yates, S. R. Quint, and H. T. Nagle: IEEE Trans. Biomed. Eng. 37 (1990) 85.

6 N. Akshay, N. A. V. Jonnabhotla, N. Sadam, and N. D. Yeddanapudi: 2010 Int. Conf. Electronics and Information Engineering (ICEIE) 2 (2010) V2-438.

7 J. C. Hsieh, W. C. Tzeng, Y. C. Yang, and S. M. Shieh: Computers in Cardiology (IEEE, 2005) 751-754.

8 Y. F. Tan and L. Du: WRI World Congr. Software Engineering 2009, WCSE'09 4 (2009) 515.

9 A. Ebrahimzadeh and M. Azarbad: 6th Int. Conf. Digital Content, Multimedia Technology and its Applications (IDC) (IEEE, 2010) 250-254.

10 H. H. So and K. L. Chan: Proc. 19th Annu. Int. Conf. IEEE Engineering in Medicine and Biology Society 1 (1997) 289.

11 M. G. Carey, S. S. Al-Zaiti, and R. A. Butler: Computing in Cardiology (IEEE, 2010) 685-688.

12 K. M. Chang: 2010 Int. Conf. Machine Learning and Cybernetics (ICMLC) 1 (2010) 210.

13 Y. H. Lee, H. W. Tseng, Y. D. Liao, T. W. Lin, and Y. L. Chen: Int. Conf. Advanced Materials for Science and Engineering (ICAMSE) (IEEE, 2016) 259-262.

14 H. W. Tseng, C. D. Huang, L. Y. Yen, T. W. Lin, Y. W. Lee, and Y. L. Chen: IEEE Int. Conf. Consumer Electronics-Taiwan (ICCE-TW) (IEEE, 2016) 1-2.

15 H. W. Tseng, Y. H. Lee, C. D. Huang, and H. Y. Huang: IEEE Int. Conf. Consumer Electronics-Taiwan (ICCETW) (IEEE, 2015) 106-107.

16 H. W. Tseng, L. P. Chin, C. D. Huang, Y. H. Lee, and F. Di: IEEE Int. Conf. Consumer Electronics-Taiwan (ICCE-TW) (IEEE, 2015) 104-105.

17 Y. H. Lee, H. W. Tseng, C. D. Huang, Y. W. Lee, and C. S. Hung: IEEE Int. Conf. Consumer ElectronicsTaiwan (ICCE-TW) (IEEE, 2015) 108-109.

18 B. A. Rajoub: IEEE Trans. Biomed. Eng. 49 (2002) 355.

19 C. L. Chang, K. P. Lin, T. H. Tao, T. Kao, and W. H. Chang: Proc. 20th Annu. Int. Conf. Engineering in Medicine and Biology Society (IEEE, 1998) 101-103.

20 L. Burattini, G. Bellagamba, F. Balestrini, N. Fratalocchi, L. Pennacchietti, and J. De Bie: Computers in Cardiology (IEEE, 2000) 367-373

21 L. Johannesen, U. S. L. Grove, J. S. Sørensen, M. L. Schmidt, J. P. Couderc, and C. Graff: Computing in Cardiology (IEEE, 2010) 979-982.

22 K. P. Lin and W. H. Chang: IEEE 17th Annu. Conf. Engineering in Medicine and Biology Society 1 (1995) 183.

23 N. M. Saad, A. R. Abdullah, and Y. F. Low: 4th Student Conf. Research and Development (SCOReD) (IEEE, 2006) 61-65.

24 R. Pratap: Getting Started with MATLAB 5-A Quick Introduction for Scientists and Engineers (Oxford University Press, 1998) p. 240.

\section{About the Authors}

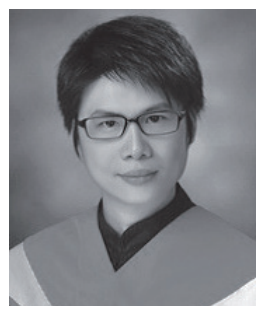

Hsien-Wei Tseng received his B.S. degree in electrical engineering from National Kaohsiung University of Applied Sciences in 2002 and obtained his Ph.D. degree in Electrical Engineering, at Tamkang University in 2010. He joined the School of Information Engineering, Longyan University, Longyan, Fujian, China, in August 2017 and now serves as a professor. His main research interests include system design and performance evaluation in wireless communication systems. 


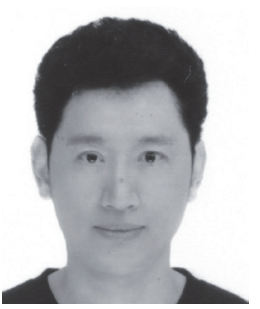

Yang-Han Lee received his B.S., M.S., and Ph.D. degrees in electrical engineering from National Taiwan University, Taipei, in 1987, 1989, and 1991, respectively. He joined the faculty of the Department of Electrical Engineering, Tamkang University, Taipei, in 1994, and now serves as a professor. His main research interests include optical fiber communication systems and communication electronics.

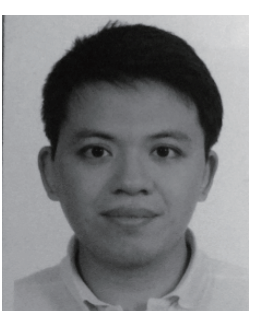

Chien-Da Huang received his B.S. and M.S. degrees in electrical engineering from Tamkang University, Taipei, in 2009 and 2011, respectively. $\mathrm{He}$ is currently a Ph.D. candidate in the Department of Electrical and Computer Engineering, Tamkang University. His main research interests include circuit design and health medical electronics.

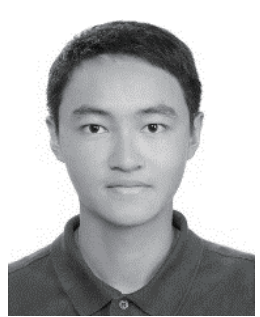

Yi-Lun Chen received his B.S. and M.S. degrees in electrical engineering from Tamkang University, Taipei, in 2015 and 2017, respectively. He is currently a Ph.D. student in the Department of Electrical and Computer Engineering, Tamkang University. His main research interests include signal processing and communication systems. 